\title{
Concentración letal 50 a 96 horas de eugenol en cachama blanca (Piaractus brachypomus)
}

\author{
Lethal (96hLC50) concentration of eugenol in the Red-bellied \\ Pacu (Piaractus brachypomus)
}

\author{
Concentração letal 50 a 96 horas de eugenol em cachama branca \\ (Piaractus brachypomus)
}

\author{
Gira A. Marín - Mendez ${ }^{1}$, Anyi Torres - Cortes ${ }^{1}$, Leidy Naranjo -Suarez', \\ Roberto A. Chacón - Novoa ${ }^{1}$, lang S. Rondón - Barragan ${ }^{2 *}$

\footnotetext{
1 Est MVZUniversidad del Tolima, Facultad de Medicina Veterinaria y Zootecnia - Departamento de Sanidad Animal

$2^{*}$ MVZ, MSc, Grupo de Investigación en Inmunología y Fisiopatología Animal - IFA; Tolima, Colombia.

Email: marinmendezgira@gmail.com
}

Recibido: julio 29 de 2011

Aceptado: julio 11 de 2012

\begin{abstract}
Resumen
El uso de anestésicos en peces ha adquirido una mayor relevancia debido al rol que desempeñan en el desarrollo de buenas prácticas de manejo, así como en la disminución de los niveles de estrés por manipulación tanto en explotaciones dedicadas a la producción de peces ornamentales como de consumo. Existen anestésicos convencionales implementados en peces tales como 2-fenoxietanol, Benzocaína, Quinaldina y MS222. No obstante, su costo y margen de seguridad en ocasiones limitan su uso. Por este motivo se requieren nuevos compuestos para el manejo adecuado de los peces, que garanticen su seguridad. El objetivo de esta investigación fue establecer la concentración letal $50\left(\mathrm{CL}_{50}\right)$ a 96 horas del eugenol (aceite de clavo derivado de Eugenia caryophyllata) en cachama blanca. Para la determinación de la $\mathrm{CL}_{50}$ se utilizaron 90 alevinos de cachama blanca (Piaractus brachypomus) de $3.5 \pm 0.5 \mathrm{~g}$ en acuarios de vidrio con un volumen cercano a los 30 litros, los parámetros de calidad del agua estuvieron dentro del rango confort para la especie. Los peces fueron expuestos a siete concentraciones de eugenol $(8,12,15,18,25,35,45 \mathrm{mg} / \mathrm{L})$ adicionalmente se evalúo un grupo control y un grupo con el diluyente anestésico por un periodo de 96 horas, en el cual se evaluó su comportamiento: eje de nado, tipo de nado y distribución en el acuario. El valor de la $\mathrm{CL}_{50}$ se estimó mediante el uso del software Trimmed Spearman-Karber y fue de $18.2 \mathrm{mg} / \mathrm{L}(p<0.05)$, acorde a lo descrito para algunas especies dulceacuícolas.
\end{abstract}

Palabras clave: anestesia, cachama blanca, $\mathrm{CL}_{50}$, eugenol.

\begin{abstract}
Anaesthetic use in fish-farming has gained in significance due to its role in good handling practice, as well as decreased stress levels when handling both ornamental fish and fish for human consumption. Conventional anaesthetics have been used in fish, such as 2-phenoxyethanol, benzocaine, quinaldine and tricaine me thanesulphonate (MS-222); nevertheless, the ir cost and safety margin have limited their use. New and safe compounds are required for suitable, safe fish handling. This study was aimed at determining the 96 -hour $50 \%$ lethal concentration $\left(96 \mathrm{hLC} 50_{50}\right)$ of eugenol (Eugenia caryophyllata
\end{abstract}


- derived clove oil) in Red-bellied Pacu. Ninety Red-bellied Pacu (Piaractus brachypomus) alevins weighing $3.5 \pm 0.5$ g body weight were used for assessing $\mathrm{LC}_{50}$; they were placed in 30-L glass aquariums where water quality came within the range of comfort for this specie. The fish were exposed to seven concentrations of eugenol $(8,12,15,18,25,35,45 \mathrm{mg} / \mathrm{L})$ for a 96-h period, as well as a control group and another group with solvent anaesthetic, during which the fishes' behaviour regarding their swimming axis, type of swimming and distribution in the aquarium was assessed. The $\mathrm{LC}_{50}$ valuewas calculated by using Trimmed Spearman-Karber (TSK) software, giving $18.2 \mathrm{mg} / \mathrm{L}(p<0.05)$, thereby agreeing with reports regarding other freshwater fish species.

Keywords: anaesthesia, Red-bellied Pacu, LC 50 , eugenol.

\begin{abstract}
Resumo
O uso de anestésicos em peixes adquiriu uma maior relevância devido ao papel que desempenham no desenvolvimento de boas práticas de manejo, bem como na diminuição dos níveis de estresse por manipulação tanto em explorações dedicadas à produção de peixes ornamentales como de consumo. Existem anestésicos convencionais implementados em peixes tais como 2-fenoxietanol, Benzocaína, Quinaldina e MS222. Não obstante, seu custo e margem de segurança em ocasiões limitam seu uso. Por este motivo se requerem novos compostos para o manejo adequado dos peixes, que garantam sua segurança. O objetivo desta investigação foi estabelecer a concentração letal $50\left(\mathrm{CL}_{50}\right)$ a 96 horas do eugenol (azeite de prego derivado de Eugenia caryophyllata) em cachama branca. Para a determinação da $\mathrm{CL}_{50}$ se utilizaram 90 alevinos de cachama branca (Piaractus brachypomus) de $3.5 \pm 0.5 \mathrm{~g}$ em aquários de vidro com um volume próximo aos 30 litros, os parâmetros de qualidade do água estiveram dentro da casta conforto para a espécie. Os peixes foram expostos a sete concentrações de eugenol $(8,12,15,18,25,35,45 \mathrm{mg} / \mathrm{L})$ adicionalmente se avalio um grupo controle e um grupo com o diluyente anestésico por um período de 96 horas, no qual se avaliou seu comportamento: eixo de nado, tipo de nado e distribuição no aquário. $\mathrm{O}$ valor da $\mathrm{CL}_{50}$ se estimou mediante o uso do software Trimmed Spearman-Karber e foi de $18.2 \mathrm{mg} / \mathrm{L}(p<0.05)$, conforme ao descrito para algumas espécies dulceacuícolas.
\end{abstract}

Palavras- chave: anestesia, cachama branca, $\mathrm{CL}_{50}$, eugenol.

\section{Introducción}

Los anestésicos desempeñan un papel importante en acuicultura al ser empleados para la realización de procedimientos médicos, quirúrgicos y experimentales, incluyendo la morfometría en peces (Hajek et al. 2006). Este tipo de prácticas generan alteraciones en el desempeño fisiológico y productivo de los animales debido a una elevación en los niveles de estrés que a su vez puede ocasionar inmunosupresión e incluso la muerte (Olivera et al. 2008). Por este motivo la implementación de agentes anestésicos se constituye en una alternativa eficiente para mitigar los efectos de la manipulación.

Dentro de los anestésicos de uso frecuente en peces se incluyen: sulfato de quinaldina, tricainametanosulfonato (MS222), 2-fenoxietanol, benzocaína, entre otros. Sin embargo, algunos de estos compuestos son considerados tóxicos para algunas especies acuáticas (Roubach et al. 2005). Actualmente, el único anestésico aprobado por Food and Drug Administration (FDA) y Environmental Protection Agency (EPA) es el MS222 para ser utilizado en peces de consumo (Velasco-Santamaría et al. 2008). No obstante, se ha demostrado que el MS222 eleva los niveles de cortisol en plasma de peces completamente anestesiados (Coyle et al. 2004). Alternativamente se han descrito técnicas de anestesia física que incluyen el uso de electricidad (electronarcosis), gas a presión o bajas temperaturas (Renault et al. 2011).

Dentro de los anestésicos no convencionales se encuentra el eugenol el cual ha sido probado en peces de agua dulce, marinos y moluscos y demostró alta efectividad y seguridad (Munday \& Wilson, 1997; Keene et al. 1998; Taylor \& Roberts, 1999; Griffiths, 2000; Lellis et al. 2000; Prince \& Powell, 2000; Durville \& Collet, 2001; Woody et al. 2002; Pirhonen \& Schreck, 2003; Seol et al. 2007). Este derivado fenólico posee una consistencia liquida - aceitosa, poco soluble en agua y soluble en alcohol (Skinner, 1994). El eugenol $\left(\mathrm{C}_{10} \mathrm{H}_{12} \mathrm{O}_{2}\right)$ se obtiene de hojas, flores, tallos y brotes de árboles de clavo (Eugenia caryophyllata y Eugenia aromatica) (Soto \& Burhanuddin, 1995). Pirhonen \& Schreck, (2003) reportan en trucha arcoiris (Oncorhynchus mykiss) disminución del consumo de alimento 4 horas posteriores a la anestesia con MS-222 y aceite de clavo. Sumado a esto algunos autores han descrito que a temperaturas altas se induce un período de recuperación más largo y, potencialmente, reduce el margen de seguridad (Hikasa et al.1986).

El presente estudio fue realizado para determinar la concentración letal 50 del eugenol en cachama blanca (Piaractus brachypomus) expuestas durante 96 horas. 


\section{Metodología}

\section{Localización}

El experimento se llevó a cabo en el Laboratorio de Toxicología de la Universidad del Tolima, adscrito a la Facultad de Medicina Veterinaria y Zootecnia, en la ciudad de Ibagué, capital del departamento del Tolima, localizada a 1.248 m.s.n.m. Con una temperatura promedio anual de $22^{\circ} \mathrm{C}$, precipitación anual de $\mathrm{mm}$ y una humedad relativa del $50 \%$.

\section{Animales}

Para los ensayos preliminares así como para el experimental se utilizaron alevinos de cachama blanca (Piaractus brachypomus) de $3.5 \pm 0.5$ g, obtenidos del Instituto de Acuicultura de los Llanos (IALL), criados en estanques de tierra y provenientes de un mismo desove.

Los animales fueron aclimatados por un periodo de 15 días bajo las condiciones de laboratorio y fueron alojados en acuarios de vidrio con un volumen cercano a los 30 litros, con aireación constante sin filtro. Durante el periodo de aclimatación los peces fueron alimentados con concentrado comercial (30\% de proteína) con una ración correspondiente al $2 \%$ de la biomasa total. Los procedimientos fueron de carácter no invasivo y aprobados por el Comité de Ética del centro de Investigaciones de la Universidad del Tolima.

Diariamente, fueron medidos los parámetros de calidad de agua correspondientes a: $\mathrm{pH}$, sólidos disueltos, temperatura ( $\mathrm{pHMeter} \mathrm{Multiparametric} \mathrm{Hanna} \mathrm{Instru-}$ ments ${ }^{\circledR}$ ) y oxigeno disuelto (OXXI 330i/set Germany).

\section{Sustancias experimentales}

Se utilizó Eugenol ${ }^{\circledR}$ (The J Bird Moyer Co., Inc, USA.), (4-alil, 2-metoxifenol), obtenido de una casa comercial, (densidad de $1.04 \mathrm{~g} / \mathrm{mL}(\sim 1000 \mathrm{mg} / \mathrm{mL})$. Para el uso como anestésico se realizó una dilución de 1:9 (v/v) con etanol para lograr una concentración final de 100 $\mathrm{mg} / \mathrm{mL}$ de eugenol en la solución stock.

\section{Diseño experimental}

Para determinar la $\mathrm{CL}_{50-96}$, se utilizaron 90 alevinos que fueron expuestos a 7 concentraciones de eugenol (8, $12,15,18,25,35,45 \mathrm{mg} / \mathrm{L})$ y un grupo control, así mismo un grupo de peces fueron sometidos durante todo el periodo experimental al diluyente del anestésico (etanol al 99.5\% disuelto en agua) para discriminar su posible efecto anestésico o tóxico. En cada una de las concentraciones experimentales se utilizaron 10 animales incluyendo el grupo control y el grupo con diluyente durante 96 horas. Se evaluó el comportamiento de los peces desde el momento en que se introdujeron al acuario con su respectivo tratamiento. Durante el periodo experimental, se manejó un modelo semiestático con recambio diario del $50 \%$ del contenido del acuario para evitar cambios en la concentración del anestésico por evaporación. Los experimentos fueron realizados siguiendo los lineamientos descritos por Organisation for Economic Co-operation and Development (OECD, 1992).

\section{Concentración letal $50\left(C_{50}\right)$}

Para determinar la concentración letal 50 se utilizó el software Trimmed Spearman-Karber), basado en la mortalidad acumulada a las 96 horas (Hamilton et al. 1977) con nivel de confianza de $p<0.05$.

\section{Resultados}

Los animales sometidos a las diferentes concentraciones de eugenol presentaron frecuentemente episodios de nado con hiperactividad esporádica y no se evidenció un patrón específico de distribución en los tercios de los acuarios. Los animales sometidos al solvente (i.e. etanol) no evidenciaron comportamientos anormales, ni signos compatibles con algún estado anestésico o de toxicidad. Los patrones de comportamiento observados se caracterizaron por la pérdida del eje de nado, nado en espiral y nado hacia la superficie a concentraciones bajas $(8,12$, $15,18 \mathrm{mg} / \mathrm{L}$ ), mientras que en las concentraciones altas $(25,35,45 \mathrm{mg} / \mathrm{L})$ se observó una rápida inducción a la anestesia y disminución pronunciada hasta el cese de los movimientos operculares hasta producirse la muerte.

El valor de la $\mathrm{CL}_{50-96}$ del eugenol en alevinos de cachama blanca corresponde a $18.2 \mathrm{mg} / \mathrm{L}(p<0.05)$. Para las concentraciones de 25,35 y $45 \mathrm{mg} / \mathrm{L}$, se presentó una mortalidad del $100 \%$ de los sujetos experimentales a las 24 horas de exposición. Mientras que en la concentración de $18 \mathrm{mg} / \mathrm{L}$ se registró una mortalidad del $60 \%$. En las concentraciones menores a $18 \mathrm{mg} / \mathrm{L}$ no se evidenció mortalidad (Figura 1).

\section{Discusión}

El eugenol como sustancia anestésica en peces requiere estudios de toxicidad que permitan inferir en cada especie en particular su margen de seguridad y validen su uso en prácticas de acuicultura tales como desove, 


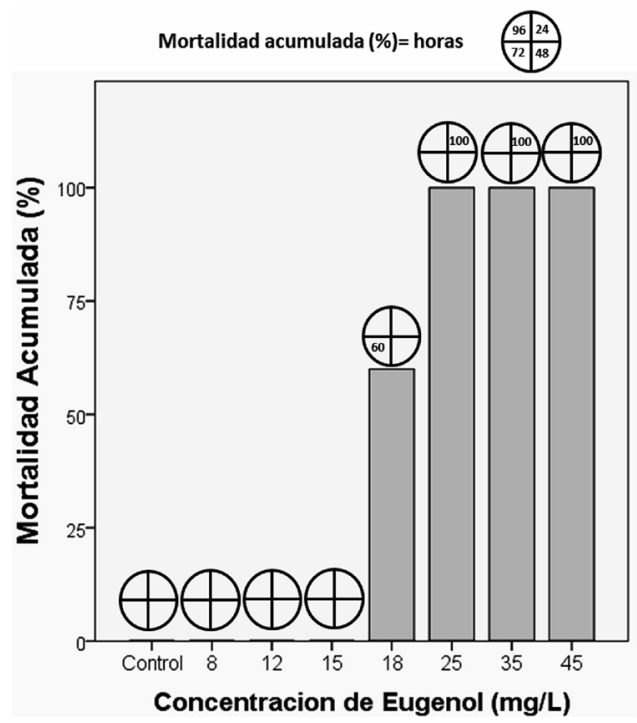

Figura 1. Porcentaje de mortalidad acumulada a las 96 horas a diferentes concentraciones de Eugenol. Cada cuadrante de la circunferencia representa el porcentaje de mortalidad registrado durante en el periodo correspondiente de exposición.

pesaje, morfometría, intervenciones quirúrgicas, toma de biopsias, entre otras.

Aunque se han adelantado estudios relacionados con la farmacodinamia del eugenol en peces y otras especies (Guénette et al. 2007ab), el mecanismo de acción aún está en investigación. Estudios in vitro han demostrado la interacción del eugenol bloqueando los receptores vaniloides (TRPV1- Transient receptor potential vanilloid 1) inhibiendo así la transmisión del dolor (Yang et al. 2003). El eugenol también inhibe el receptor N-metil D-aspartato (NMDA) del gutamato y es agonista del neurotransmisor acido gamma aminobutírico (GABA) de acuerdo a lo reportado por Sladky et al. (2001), que causan depresión del Sistema Nervioso Central (SNC) y a esto se debe su efecto anestésico, por ende su toxicidad puede estar mediada por la fuerte depresión del centro respiratorio (Guénette et al. 2007b).

La evaluación de la toxicidad aguda del eugenol ha sido descrita en diferentes especies de organismos acuáticos. Así, en invertebrados acuáticos como el caracol de agua dulce Biomphalaria alexandrina se registra una $\mathrm{CL}_{50}$ de $28 \mathrm{mg} / \mathrm{L}$ (Din, 2006) y en algunos artrópodos de $74.5 \mu \mathrm{g} / \mathrm{mL}$ (Sampaio et al. 2009).

Para peces de agua dulce se ha descrito la $\mathrm{CL}_{50}$ del eugenol que oscila entre 14 y $60 \mathrm{mg} / \mathrm{L}$. En este trabajo se determinó la $\mathrm{CL}_{50-96}$ de eugenol en $18.2 \mathrm{mg} / \mathrm{L}$ en alevinos de cachama blanca, lo cual concuerda con lo reportado para bagre europeo (Silurus glanis L.) 18.4 mg/L (Velisek et al. 2006), 18.1 mg/L en carpa común
(Cyprinus carpio L.) y en trucha arcoíris (Oncorhynchus mykiss) de $14.1 \mathrm{mg} / \mathrm{L}$ (Velisek et al. 2005). Acorde con lo hallado en el presente estudio, Soto \& Burhanuddin (1995) encontraron que en el pez conejo de mancha dorada (Siganus guttatus se inicia la muerte a las 20 horas de exposición a $15 \mathrm{mg} / \mathrm{L}$ de eugenol. De la misma manera, Charoendat et al. (2009), describen la $\mathrm{CL}_{50}$ a 24 horas del eugenol en $16.98 \mathrm{mg} / \mathrm{L}$ para alevinos de tilapia nilótica (Oreochromis niloticus). No obstante, Gullian \& Villanueva (2009) reportan la $\mathrm{CL}_{50}$ de 60 $\mathrm{mg} / \mathrm{L}$ en juveniles de cobia (Rachycentron canadum) que difiere considerablemente de lo encontrado en este estudio.

\section{Agradecimientos}

A los miembros del Grupo de Inmunología y Fisiopatología Animal (IFA) por el acompañamiento en las labores diarias del sostenimiento de la fase experimental, al personal del Laboratorio de Toxicología y del Laboratorio de Diagnóstico Veterinario de la Universidad del Tolima por permitir el establecimiento del experimento y el apoyo constante de este.

\section{Referencias}

Barbosa L, Moraes G, Kiochi L. Respostas metabólicas do matrinxã submetido a banhos anestésicos de eugenol. Acta Scientiarum Biological Sciences, 2007; 29 (3): 255-260.

Charoendat U, Areechon N, Srisapoome P, Chantasart D. Efficacy of synthetic eugenol as an anesthetic for nile tilapia (Oreochromis niloticus Linn.). Kasetsart Journal: Natural Sciences, 2009; 43(5): 132-140. 
Coyle S, Durborow R, Tidwell J. 2004. Anesthetics in aquaculture. Southern Regional Aquaculture Center, Publication No. 3900, Texas.

Deriggi G, Kiochi L. Stress responses to handling in Nile tilapia (Oreochromis niloticus Linnaeus): assessment of eugenol as an alternative anesthetic. Acta Scientiarum Biological Sciences, 2006; 28(3): 269-274.

Din AT. Molluscicidal effect of three monoterpenes oils on schistosomiasis and fascioliasis vector snails in Egypt. Journal of the Egyptian Society of Parasitology, 2006; 36(2): 599-612.

Guénette S, Ross A, Marier J, Beaudry F, Vachon P. Pharmacokinetics of eugenol and its effects on thermal hypersensitivity in rats. European Journal of Pharmacology, 2007a; 562: 60-67.

Guénette S, Uhland F, Hélie P, Beaudry F, Vachon P. Pharmacokinetics of eugenol in rainbow trout (Onchorhynchus mykiss). Aquaculture, 2007b; 266: 262-265.

Gullian M, Villanueva J. Efficacy of tricaine methanesulphonate and clove oil as anaesthetics for juvenile cobia Rachycentron canadum. Aquaculture Research, 2009; 40(7): 852-860.

Hajek G, Kyszejko B, Dziaman R. The anaesthetic effect of clove oil on common carp, Cyprinus carpio L. Acta Ichthyologica Et Piscatoria, 2006; 36(2): 93-97.

Hamilton M, Russo R, Thurston R. Trimmed Spearman-Karber method for estimating median lethal concentrations in toxicity bioassays. Environmental Science \& Technology, 1977; 11: 714-719.

Hikasa Y, Takase K, Ogasawara T, Ogasawara S. Anesthesia and recovery with tricaine methanesulfonate, eugenol and thiopental sodium in the carp, Cyprinus carpio. Japonese Journal of Veterinary Science, 1986;48: 341-351.

OECD. 1992. Fish, Acute Toxicity Test. Guideline for testing of chemicals No 203. Adopted by the Council on $17^{\text {th }}$ July.

Olivera L, Branco C, Luscher A, Danile de Lira A, Rocha T, et al. Eugenol como anestésico para tilápia-do-nilo. Pesquisa Agropecuária Brasileira, 2008; 43(8): 1069-1074.

Pirhonen J, Schreck C. Effects of anaesthesia with MS-222, clove oil and $\mathrm{CO}_{2}$ on feed intake and plasma cortisol in steelhead trout (Oncorhynchus mykiss). Aquaculture, 2003; 220: 507-514.
Renault S, Daverat F, Pierron F, Gonzalez P, Dufour S, Lanceleur L, Schäfer J, Baudrimount $M$. The use of Eugenol and electro-narcosis as anaesthetics: Transcriptional impacts on the European eel (Anguilla anguilla L.). Ecotoxicology Environmental Safety, 2011; 74 (6): 1573-1577.

Roubach R, Carvalho L, Leao F, Vall A. Eugenol as an efficacius anaesthetic for tambaqui Colossoma macropomun (Cuvier). Aquaculture Research, 2005;36: 1056-1061.

Sampaio C, Ferreira Y, Sousa M, Bandeira P, Albuquerque M, et al. 2009. Atividade larvicida contra o Aedes aegypti e bactericida dos óleos essenciais de Croton zehntneri Pax. \& K.Hoffm var. Estragol e eugenol. 32a Reunião Anual da Sociedade Brasileira de Quimica.

Sladky K, Swanson C, Stoskopf M, Loomis M, Lewbart G. Comparative efficacy of tricaine metanesulfonate and clove oil for use as anesthetics in red pacu (Piaractus brachypomus). American Journal of Veterinary Research, 2001;62 (3): 337 - 342.

Soto C, Burhanuddin. Clove oil as a fish anaesthetic for measuring length and weight of rabbitfish (Siganus lineatus). Aquaculture, 1995; 136: 149-152.

Velasco Y, Palacios C, Cruz P. Eficiencia anestésica de 2-fenoxietanol, benzocaina, quinaldina y metasulfonato de tricaina en alevinos y juveniles de cachama blanca (Piaractus brachypomus). Revista MVZ Córdoba, 2008;13(3): 1435-1445.

Velisek J, Svodová Z, Piackvova V, Groch L, Nepejchalova L. Effects of clove oil on common carp (Cyprinus carpio L.). Veterinary Medicine Czech, 2005; 50(6): 269-275.

Velišek J, Svodova Z, Piackvova V. Effects of clove oil anaesthetic on rainbow trout (Onchorynchus mykiss). Acta Veterinaria Brno, 2005; 74: 139-146.

Velî́sek J, Wlasow T, Gomulka P, Svobodová Z, Novotný L, Ziomek E. Effects of clove oil anaesthesia on european catfish (Silurus glanis L.). Acta veterinaria Brno, 2006; 75: 99-106.

Yang B, Piso Z, Dim Y, Lee C, Park K, et al. Activation of vanilloid receptor 1 (VR1) by eugenol. Journal of Dental Research, 2003, 82: 781-785. 